\title{
SIMULATING ENERGY CONSUMPTION OF AUXILIARY UNITS IN HEAVY VEHICLES ${ }^{1}$
}

\author{
Niklas Pettersson, Karl Henrik Johansson
}

\author{
Department of Signals, Sensors \& System, Royal Institute of Technology, Sweden \\ niklas.pettersson@s3.kth.se, kallej@s3.kth.se
}

\begin{abstract}
Models that can be used to analyse the fuel saving potential of electrically driven auxiliaries in heavy vehicles are presented. With the purpose of evaluating the influence on fuel consumption from various concepts and control principals, a model library is developed in the modelling language Modelica. The library contains a mixture of models developed from physical principles and models fitted to collected data. Modelling of the cooling system is described in some detail. Simulation results are compared with measurement data from tests in a wind tunnel. Copyright $@ 2003$ IFAC
\end{abstract}

Keywords: Automotive Control, Energy Management Systems, Computer Simulation, Modelica.

\section{INTRODUCTION}

Improving fuel efficiency is central when developing heavy vehicles. The cost for fuel constitutes a major part of the total costs for transport of goods. Thus, a fuel-efficient truck will be economically attractive to own and can be sold at a higher price. In the same time, fuel efficiency is directly related to the environmental impact of road transports. More and more focus is put on environmental issues, and in the end, only the ones that can adapt to the demands on reduced environmental impact from their business will survive. Over the years, huge efforts have been put on improving efficiency of the combustion engine. However, the marginal cost for achieving a certain improvement of the engine is increasing. Therefore, it is getting increasingly more rewarding spending development effort on optimising the efficiency of the auxiliary units and subsystems in the vehicle. Resent advances within the area of electrical drive systems, and alternative power production devices such as fuel cells, may offer a possibility to utilise electricity powered auxiliaries where it former was non-practicable or noneconomical. In the process of introducing new technologies, working with models and computer simulations is essential. Simulations studies are needed both to evaluate novel concepts and to increase the knowledge of how the behaviour of today's design.

This paper presents the work of developing vehicle models that can be used to evaluate alternative architectures for the electrical system in heavy vehicles. With aid of the simulation models, the potential energy savings of new designs can be assessed. Here the ideas behind development and maintenance of a comprehensive model library are presented. The Modelica language is used to build

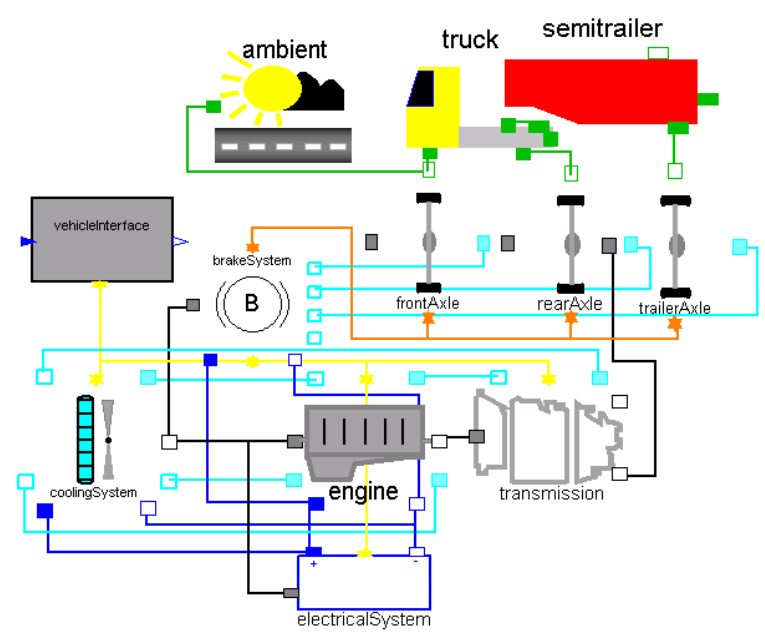

Fig 1. Modules of the simulation model.

models with a modular structure. Figure 1 shows composition of the model at the highest level.

In the simulations, the vehicle is set to drive a road with varying topology and speed limit that have been obtained from recordings of real roads. The vehicle is assumed to run on cruise control and with computer-controlled gear shifting (automated manual transmission). Algorithms from the production version of the control are incorporated in the simulation model. The vehicle model has been validated with respect to the energy consumption of the combustion engine and losses such as rolling resistance and air drag, (Sandberg, 2001). Influences from the, sub-systems, the cooling system, and the electrical network, were only included as a lumped effect on the net fuel consumption. This work refines the description of the auxiliary units. The paper describes the modelling of the cooling system in some detail. Sub-models are built from physical principles, resulting in grey-box models with

\footnotetext{
${ }^{1}$ This work is supported by Scania CV AB and Vinnova.
} 
parameters identified from various tests in a laboratory environment. The sub-models are assembled into a model of the complete vehicle. Measurements collected from tests in a wind tunnel are used to tune the performance of the total model. The model is validated against data recorded from a dynamic driving cycle in the wind tunnel.

\section{SELECTION OF MODEL STRUCTURE}

The prime goal with the vehicle model is to serve as a tool for study effects on the fuel economy from different designs of sub-systems. The main quantity that is studied is flow of energy between the parts of the vehicle. To give an accurate estimate of the energy balance, the model must cover the whole vehicle and describe processes involved in the energy conversion with a significant level of detail. Besides description of physical phenomena, it contains control software and various look-up tables. We refer to this type of model as simulation models since it (in contrast to simpler models of sub-systems or blackbox models of complete processes) might not be possible to summarise the model in a set of mathematical equations. In the literature this type of models sometimes are referred to as software models (cf., Ljung, 1999). Although the model might be comprehensive, by necessity it must in many parts be a rough approximation in order to keep the complexity on a reasonable level. Fast dynamics and other effects not relevant for the study may be neglected.

Models used in vehicle development can be classified in three groups according to which level of detail they use to describe the physical world, shown in figure 2. The most detailed models, referred to as special purpose models, are used designing hardware components. In this category one find models of the combustion process, 3-D models of the suspension etc. On the other extreme we find models used for control design. Since their limited extent allows for a mathematical description we denote these models mathematical models. Often mathematical models represent an abstract image of the physical world. Parts may be lumped into a single quantity, e.g., total inertia felt in one motion, blurring out the interfaces between components in the system described in the model. Similarly to special purpose models, mathematical models typically cover a limited part of the vehicle.

\subsection{Simulation models}

It has become more and more important to perform simulations covering the complete behaviour of the vehicle as control functions develop to be more integrated. Beside this, overall performance parameters as fuel consumption, electric energy balance and cooling capacity have become more critical in vehicle design. Models integrating relevant descriptions of major parts of the vehicle tend to grow in complexity.

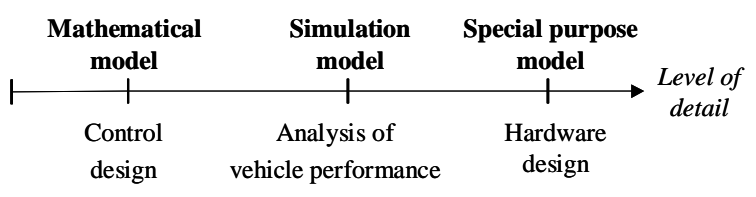

Fig 2. Classes of models used in the automotive industry.

To assemble the model in a closed form system of equation might not be possible. Stability of complex systems may not be possible to analyse mathematically. To study stability with simulation models heuristically may be the only possible alternative. In general, not only the input-output behaviour of model is of interest. Internal signals may be just as important to study. Therefore it is preferable to split up the model in components corresponding to physical objects and derive submodels from first principles. Structured in this way, simulation models are intrinsically multi-domain models.

Although all the classes of models play a specific part, it is advantageous to organise the modelling effort in an integrated way. Clearly, there exist a possibility to utilise results gained working with one type of model also for other type of models. From the perspective of simulation models, an outline of benefits with integrated modelling efforts might include: (1) time will be saved assembling a simulation models if modelling paradigms and measurement data can be adopted from the teams working with special purpose models. (2) Mathematical and simulation models can share databases for parameter values. In case either of them work with a more lumped definition of parameters, it may be possible to implement translation rules for transferring on type of parameter set to the other. (3) Control algorithms developed based on a mathematical model can be tested out together with a simulation model containing more details before the control is applied in a real vehicle. (4) In the work with complex simulation models one often have to resort to a simplified mathematical model in order to gain insight in dependencies between parts of the model or to do sensibility analysis of parameter influences. (5) The work with simulation models can give useful input to the hardware design. Results from a complete vehicle simulation can be used to define operating profiles for the components such as average speed, time spent on a certain load, etc.

\section{MODEL LIBRARY}

A library of simulation models is developed according to the principles described in the previous section. The library is developed in Modelica ${ }^{\mathrm{TM}}$, (Modelica Association, 2000). Modelica is an object oriented modelling language well suited to describe behaviour of complex systems containing parts from different engineering disciplines, e.g., mechanics and electronics. 


\subsection{Library structure.}

In contrast to the Modelica Standard Library, (Modelica Association, 2002), the library is not organised in different engineering disciplines. Instead it is organised after the parts of the truck. The library, named Scania Modelica Library, SML, consist of four main branches:
1. Interfaces
2. Components
3. Modules
4. Examples

The principal structure of the library can be viewed in figure 3.

The Interface branch contains classes describing connections between model components. Although the library relies heavily on connector classes defined in the Modelica Standard Library, some unique connectors are defined. One example is the CAN connector, used to mimic the information flow between control units in the truck. Further, under the Interfaces sub-library Media, base classes for thermodynamic and hydraulic models are found. These base classes are mainly used in models of components in the cooling system. In the thermodynamic and hydraulic base classes many of the modelling ideas used are adopted from the Modelica library ThermoFluid developed by Thummescheit, et al. (2000). However, here a somewhat simpler structure and less extensive description of media properties are used. In the Components branch models of all physical parts needed to build up the complete model of a truck are gathered. Modules, in the next branch, are a higher level of abstraction, and contain more compound models. The idea is to define a set of generic modules with well-defined interfaces that can be used to for simulations with various purposes. In the last branch a number of working examples is built that can be used directly for simulations.

Figure 4 illustrate how the models are parameterised to obtain modules that correspond to physical modules. Each component contains a placeholder for a set of parameters of a defined structure. Parameter sets with values describing various versions of the components are gathered in special sub-libraries. When modules are put together, illustrated with the cooling module, the generic placeholders are replaced with the parameter set of the current versions of components.

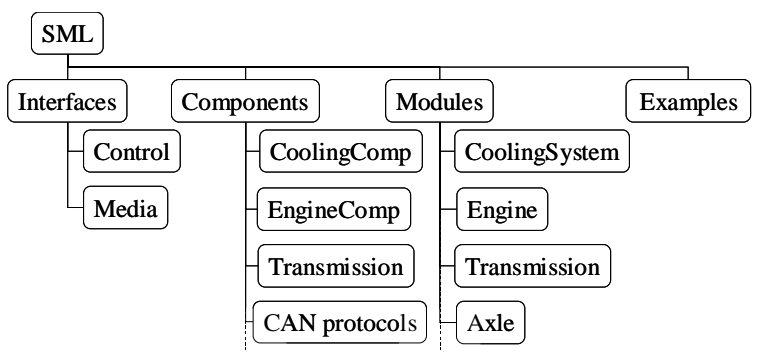

Fig. 3. Structure of the Scania Modelica Library.

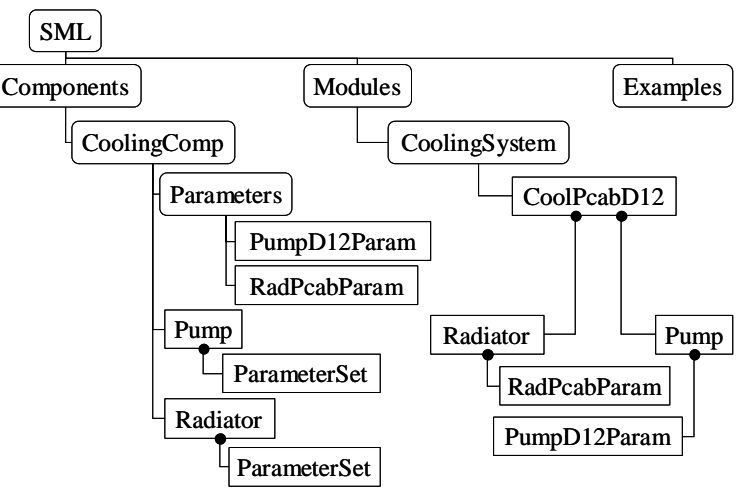

Fig. 4. Parameterisation of the model exemplified with the cooling module.

\section{COOLING SYSTEM MODULE}

The cooling system is one of the modules of the vehicle model. Energy consumers in the cooling system are primarily the cooling fan and the water pump. In heavy vehicles, these units normally are mechanically driven. The model corresponds the current design of a Scania truck where the water pump is directly driven from the crankshaft while the cooling fan is connected to the shaft via a viscous clutch enabling a passive speed control. However, the basic structure allows for changing the model to describe other ways of driving and controlling these auxiliaries.

\subsection{Cooling system components}

The main parts of the cooling system are modelled, using the thermodynamic and hydraulic base classes. In figure 5 the structure of the cooling system is depicted. The model mainly consists of two adjoining flows of mass and energy: the flow of coolant fluid and the airflow.

The pump drives the flow of coolant fluid through the engine and the retarder. The retarder is a hydraulic brake mounted on the secondary side of the gearbox. When used, it produces heat that is emitted to the cooling system. The temperature of the coolant is controlled with the thermostat by splitting up the coolant flow into one part passing the radiator and one part flowing in a by-pass pipe.

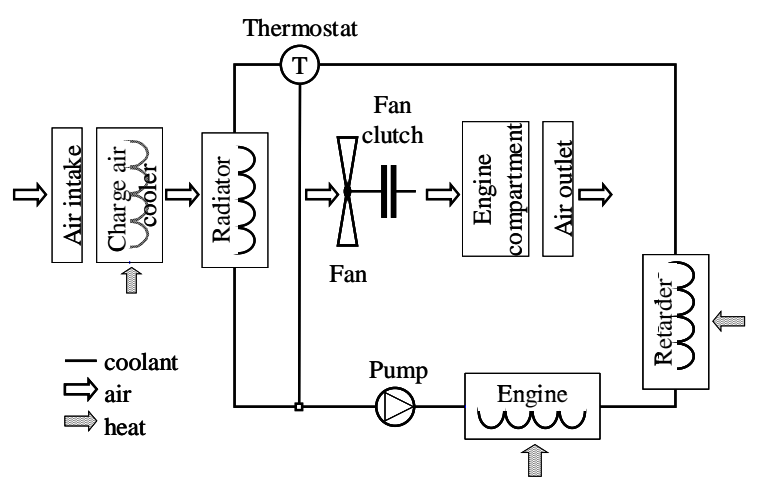

Fig. 5. Components in the cooling system module. 
The air enters in the air intake at the front of the truck $\mathrm{cab}$ and exits at the air outlet at the rear. The airflow is partly driven by the fan and partly by the pressure build up caused by the wind speed at the intake and outlet. The air is used to cool down both the turbo charged intake air to the engine, and the coolant fluid. The charge air cooler and the radiator are connected in series so that the cooling air first passes the charge air cooler and then the radiator. Both the charge air cooler and the radiator are cross directional heat exchangers, i.e., the hot and cool media streams are perpendicular to each other.

The models of the coolant and the air streams are built up with alternating control volumes and flow models. In the control volumes, mass and energy balances are defined, while in the flow models, relations between the pressure drop and the flow are determined. The control volumes describe the dynamic behaviour and are parameterised purely with geometrical quantities and properties of the contained media. The flow models describe pressure drops, heat transfer and consumed power based on empirical relations. No explicit identification of the parameters of the control volumes is needed, since they could be found in the technical specification of the components. The parameters of the flow models, however, typically have to be estimated from data.

\subsection{Dynamics of the cooling system}

For the control volumes it is possible to select which state representation that should be used. The transformation of state variables from the primary mass and energy balances to the selected states is dependent on the properties of the media inside the volume. The modelling of the control volumes is rather standard. Here it essentially follows the principles in ThermoFluid (Tummescheit et al. 2000)

For the airflow, pressure, $p$, and temperature, $T$, are chosen as state variables. The transformed balance equations then become

$$
\begin{array}{r}
m \frac{\partial u}{\partial p} \dot{p}+m \frac{\partial u}{\partial T} \dot{T}=\dot{U} \\
V \frac{\partial \rho}{\partial p} \dot{p}+V \frac{\partial \rho}{\partial T} \dot{T}+\rho \dot{V}=\dot{m}
\end{array}
$$

Here $\dot{U}$ and $\dot{m}$ denote the net flow of energy and mass into the control volume while $m$ and $V$ are the mass trapped in the volume and the size of the volume, respectively. Additionally, the air is regarded as an ideal gas yielding the following expressions for the density, $\rho$, and the partial derivatives in equation (1)

$$
\begin{aligned}
\rho & =\frac{p M}{T R} & & \\
\frac{\partial u}{\partial p} & =0, & & \frac{\partial \rho}{\partial p}=\frac{M}{T R} \\
\frac{\partial u}{\partial T} & =c_{v}, & & \frac{\partial \rho}{\partial T}=-\frac{p M}{T^{2} R}
\end{aligned}
$$

where $M$ denotes the molar mass and $c_{v}$ the specific heat capacity at constant volume, respectively, while $R$ is the molar gas constant.

Similar expressions are used for the state derivatives of the coolant media, although only the temperature is chosen as state variable. The pressure of the coolant is determined purely from static hydraulic relationships.

\subsection{Parameters of the flow models}

For the airflow, pressure drops in the components along the flow path are modelled as an exponential friction loss

$$
\Delta p=c|q| \dot{m}^{e}
$$

The frictional pressure losses in the coolant path is modelled with second order polynomials

$$
\Delta p=c_{2}|q| q+c_{1} q
$$

The pressure rise in the pump and the fan depend on the flow through the components and the angular velocity of the shaft. In the model the following equations are used to describe the operation of the pump and the fan, respectively

$$
\begin{gathered}
\Delta p=R_{1}|\omega| \omega+2 R_{2} \omega q-R_{3}|q| q \\
\Delta p=R_{1} \rho|\omega| \omega+2 R_{2} \omega \dot{m}-R_{3}|q| \dot{m}
\end{gathered}
$$

In equation (3)-(6), $q$ and $\dot{m}$ denotes volume flow rate and mass flow rate, respectively, while $\omega$ denotes the angular velocity of the pump or the fan.

The wind speed gives rise to a differential pressure at the air intake and outlet relative the ambient pressure. In the model, the pressure difference depends on the wind speed, $v$, the air density, $\rho$, and the non-dimensional coefficient $C D$ according to

$$
\Delta p=C D \frac{\rho}{2} v^{2}
$$

In order to find the parameter values of the submodels, experimental data is collected from tests on individual components in a laboratory environment. For each component, a small identification problem is solved trying to fit the predicted output from the models to the measurements. The model predictions can in the most cases be expressed as linear regressions from which the parameters estimates that give the best fit in a least square sense can be found. Essentially parameters of equation (3)-(7) and other characteristics are identified for each component depicted in the overview of the cooling module in figure 5. Table 1 summarises which parameters that are identified and what data that are used. 
Input from other parts of the total model is primarily heat losses that need to be cooled away. The engine emits heat to the cooling system both directly into the engine block, which is heated up by the combustion, and through the cooling of the charge air. The amount of heat depends on the current torque and speed of the engine. In the model this calculated from a look-up table. The table is obtained from measurements done in test cells. The heat emitted to the cooling system from the retarder is directly proportional to the braking power. In some submodels, the parameters solely represent basic quantities such as mass or volume that are found from the data sheet of the corresponding component.

The tests are performed in the laboratory under wellcontrolled conditions. As a result the obtained prediction errors are very small as can be seen by the example in figure 6 , showing the pressure drops in the airflow path.

\section{ASSEMBLING THE TOTAL MODEL}

The modelling errors in the sub-models are very small. However, when they are assembled to a full model, effects that are not handled in the sub-models may play an important role. It may be effects from the installation the truck cab such as the piping between the components. Non-linearities may amplify small errors in the sub-models when these are connected and new feedback paths are closed. It can be shown, using a simplified model of the cooling system, that the change of temperature of the coolant in steady state due to a small perturbation of the airflow is proportional to the squared inverse of the airflow. Thus, the simulated temperature will be very sensitive to modelling errors influencing the airflow.

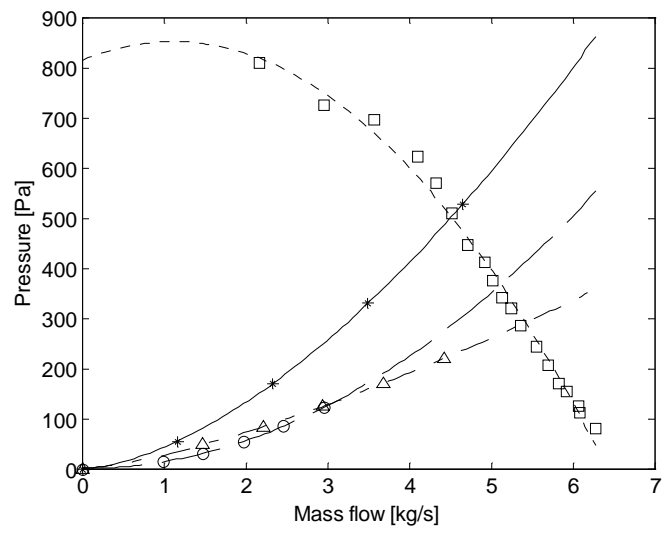

Fig. 6. Pressure drop as a function of airflow for the charge air cooler model (solid) compared with measurements (stars). Corresponding drops for radiator (dashed and triangles), and engine compartment (dash-dotted and circles).

Pressure rise of the fan model (dotted) at 1400 rpm compared with measurements (squares).
Further, for the pressure build-up due to the wind speed there exist no practicable experiment on a component level. Therefore, the result of the total model is verified through comparison with experimental data collected in a wind tunnel. In the wind tunnel, the vehicle is driven on a dynamometer with a defined load and speed of the engine. Fans are used to simulate the wind speed. Results from nine steady-state tests and two step-response tests are used to tune the model parameters. A number of the parameters in the sub-models are assigned as slack parameters that are adjusted to fit the behaviour of the total model to the measurements. In table 1 the choice of slack parameters is indicated in the last column. In figures 7 and 8 the cooling temperature obtained with the tuned model are compared with measurements.

Table 1 Summary of model components in the cooling module.

\begin{tabular}{|c|c|c|c|}
\hline Component & Characteristic & $\begin{array}{l}\text { Data } \\
\text { source }\end{array}$ & Slack \\
\hline \multirow[t]{2}{*}{ Pump } & - Pressure rise & Rig test & $\mathrm{s}$ \\
\hline & $\begin{array}{l}\text { - Power } \\
\text { consumption }\end{array}$ & Rig test & \\
\hline \multirow[t]{4}{*}{ Engine } & - Flow resistance & Rig test & \\
\hline & $\begin{array}{l}\text { - Heat } \\
\text { capacitance }\end{array}$ & $\begin{array}{l}\text { Data } \\
\text { sheet }\end{array}$ & $\mathrm{s}$ \\
\hline & $\begin{array}{l}\text { - Heat emission } \\
\text { to coolant }\end{array}$ & Rig test & \\
\hline & $\begin{array}{l}\text { - Heat emission } \\
\text { from charge air }\end{array}$ & Rig test & \\
\hline \multirow[t]{3}{*}{ Retarder } & - Flow resistance & Rig test & \\
\hline & $\begin{array}{l}\text { - Heat } \\
\text { capacitance }\end{array}$ & $\begin{array}{l}\text { Data } \\
\text { sheet }\end{array}$ & $\mathrm{s}$ \\
\hline & - Heat emission & None & \\
\hline \multirow[t]{3}{*}{ Thermostat } & $\begin{array}{l}\text { - Opening } \\
\text { characteristic }\end{array}$ & Rig test & \\
\hline & - Flow resistance & Rig test & \\
\hline & $\begin{array}{l}\text { - Dynamic } \\
\text { response }\end{array}$ & Rig test & \\
\hline \multirow[t]{4}{*}{ Radiator } & $\begin{array}{l}\text { - Flow resistance } \\
\text { coolant }\end{array}$ & Rig test & \\
\hline & $\begin{array}{l}\text { - Flow resistance } \\
\text { air }\end{array}$ & Rig test & \\
\hline & $\begin{array}{l}\text { - Operating } \\
\text { characteristics }\end{array}$ & Rig test & \\
\hline & $\begin{array}{l}\text { - Heat } \\
\text { capacitance }\end{array}$ & $\begin{array}{l}\text { Data } \\
\text { sheet }\end{array}$ & \\
\hline Air intake & $\begin{array}{l}\text { - Pressure } \\
\text { build-up }\end{array}$ & None & $\mathrm{s}$ \\
\hline $\begin{array}{l}\text { Charge air } \\
\text { cooler }\end{array}$ & - Flow resistance & Rig test & $\mathrm{s}$ \\
\hline \multirow[t]{2}{*}{ Fan } & - Pressure rise & Rig test & \\
\hline & $\begin{array}{l}\text { - Power } \\
\text { consumption }\end{array}$ & Rig test & \\
\hline Fan clutch & $\begin{array}{l}\text { - Slip } \\
\text { characteristics }\end{array}$ & Rig test & \\
\hline $\begin{array}{l}\text { Engine } \\
\text { compartment }\end{array}$ & - Flow resistance & Rig test & \\
\hline Air outlet & $\begin{array}{l}\text { - Pressure } \\
\text { build-up }\end{array}$ & None & $\mathrm{s}$ \\
\hline
\end{tabular}




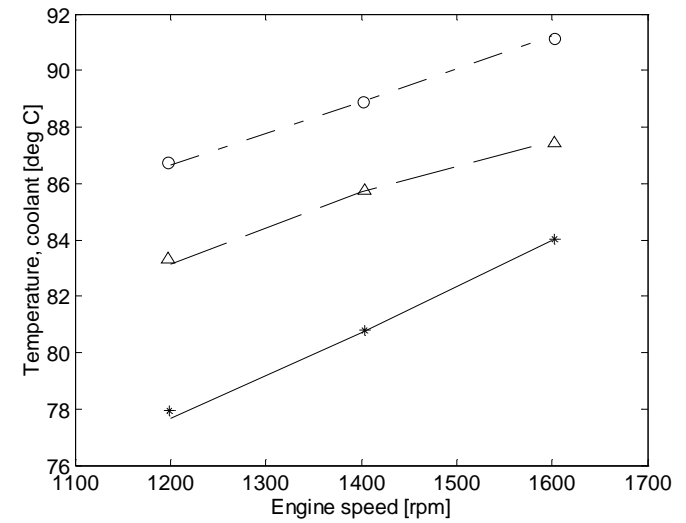

Fig. 7. Simulated temperature of the coolant in steady state at $80 \mathrm{~km} / \mathrm{h}$ with full load and different speeds on the engine (solid) compared with measurements (stars). Corresponding at 60 $\mathrm{km} / \mathrm{h}$ (dashed and triangles) and at $40 \mathrm{~km} / \mathrm{h}$ (dash-dotted and circles).

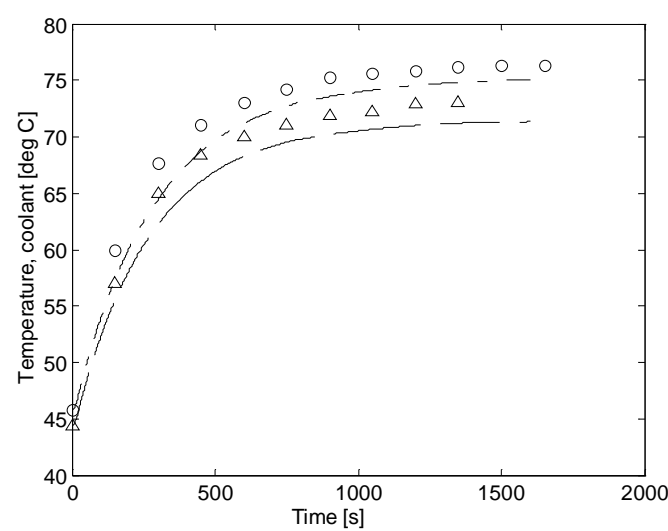

Fig. 8. Simulated response of the coolant temperature on a step in the engine load at $60 \mathrm{~km} / \mathrm{h}$ with engine speed $1400 \mathrm{rpm}$ (dashed), compared with measurements (triangles). Corresponding at $40 \mathrm{~km} / \mathrm{h}$ (dash-dotted and circles).

As a last step of the parameter identification, a validation of the total model is performed. Validation data is recorded during a dynamic drive cycle in the wind tunnel, where the load and speed of the dynamometer is programmed to follow a cycle corresponding to a specified road. In figure 9 the simulation result is compared with measurements where the dynamometer follows the profile of a $57 \mathrm{~km}$ section of the road between the cities Koblenz and Trier in Germany. The validation shows that the model is capable to capture the main dynamics of the cooling system while it does not describe the small oscillations observed in the measurements. The oscillations around $80^{\circ} \mathrm{C}$ most likely have its origin in the complex dynamics of the thermostat. The model of the thermostat is a rather rough approximation and do not give raise to corresponding oscillations around the opening temperature. Despite the observed differences, the model should be sufficient to evaluate the energy consumption of the auxiliary units in the cooling system.

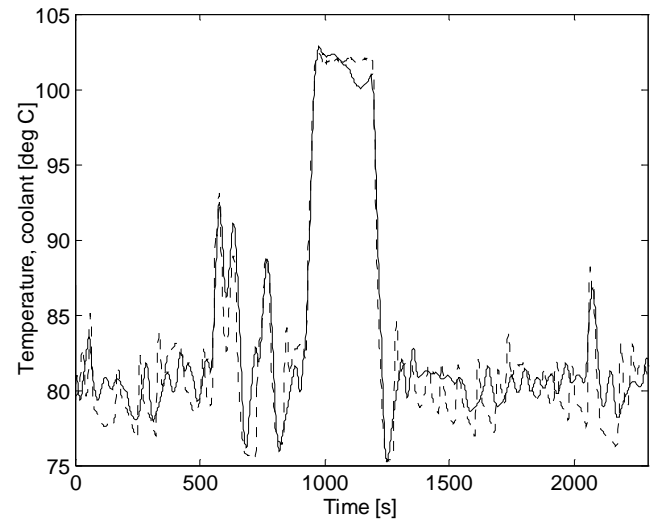

Fig. 9. Simulated coolant temperature (solid) during a dynamic driving cycle compared with measurements (dotted).

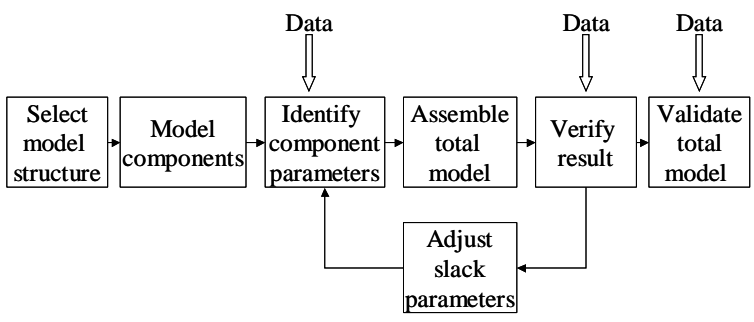

Fig. 10. Procedure of sequential modelling and identification.

\section{SUMMARY}

The procedure of building a model consisting of submodels with physical interpretation and performing sequential identification of parameters may be summarised with the illustration in figure 10 . The cascaded identification allows for keeping the physical structure of the model while the total behaviour of the model can be tuned to give a good fit to measurement data.

\section{REFERENCES}

Ljung, L., (1999). System Identification, Theory for the User. Prentice Hall, Upper Saddle River, New Jersey 07458.

Modelica Association, (2002). Modelica ${ }^{\mathrm{TM}}$ - A Unified Object-Oriented Language for Physical Systems Modeling. Language Specification Ver2.0. http://www.modelica.org/, 2002.

Sandberg, T., (2001). Heavy Truck Modeling for Fuel Consumption Simulations and Measurements. Licentiate thesis, department of Electrical Engineering, Linköping University Sweden.

Tummesheit, H., Eborn J. and Wagner FJ.

Development of a Modelica Base Library for Modeling of ThermoHydraulic Systems. Proceedings of Modelica Conference 2000. 